\title{
Sparse Reconfigurable Adaptive Filter with an Upgraded Connection Constraint Algorithm
}

\author{
Hong Chang', Suk-seung Hwang ${ }^{2}$ \\ ${ }^{1}$ Department of Advanced Parts \& Materials Engineering, Chosun University, Gwangju, 501-759, Korea \\ ${ }^{2}$ Department of Mechatronics Engineering, Chosun University, Gwangju, 501-759, Korea
}

\begin{abstract}
A sparse reconfigurable adaptive filter (SRAF) based on a photonic switch determines the appropriate time delays and weight values for an optical switch implementation of tapped-delay-line (TDL) systems. It is well known that the choice of switch delays is significantly important for efficiently implementing the SRAF. If the same values exist as calculating the sum of weight magnitudes for implementing the connection constraint required by the SRAF, conventional connection algorithm based on sequentially selection the maximum elements might not work perfectly. In an effort to increase the effectiveness of system identification, an upgraded connection algorithm used progressive calculation to obtain the better solution is considered in this paper. The performance of the proposed connection constraint algorithm is illustrated by computer simulation for a system identification application.
\end{abstract}

Keywords : Adaptive Filtering, Connection Constraint Algorithm, Adaptive Algorithm, System Identification.

\section{Introduction}

The SRAF [1] considered here was developed as a new application for a non-blocking optical switch that is based on micro-electromechanical systems (MEMS) technology [2], consisting of a large number of input and output delays connected together by weights that are computed by an adaptive algorithm. These tap weights can be represented by a sparse matrix (size $N \times N$ ) with the constraint that at most only one element in each row and column is nonzero; the nonzero weights combine the input and output delays so that up to $N^{2}$ different time delays are possible. Although the TDL filters have previously been considered for optical fiber technology, they have not worked well due to the small size of the switches [3]. Recently, large-scale three-dimensional optical switches have been developed using MEMS technology [4]-[6]. Due to MEMS technology, large optical switches can be efficiently implemented, thus broadening the range of possible application.

The SRAF uses adaptive algorithm [7] to choose the appropriate time delays and compute the weight values of the optical switch according to the specific application. In previous work[2], a cross-correlation-based (CCB) algorithm was investigated for selecting the specific switch connections and a system-based (SB) algorithm [8] which employs a system identification formulation was also be presented. The previous connection algorithm [9] based on sequentially choosing the

Manuscript received Nov. 2, 2011; revised Dec. 12, 2011; accepted Dec. 12;

${ }^{+}$Corresponding author(Suk-seung)

This study was supported by research funds from Chosun University, 2010. maximum elements might not work well when the same values exist as computing the summation of the weight values. In this case, the performance should be degraded, because some weights are randomly chosen. In order to overcome this problem, an upgraded connection algorithm used progressive computation to obtain the better solution is motivated for improving the accuracy of the system identification. In this paper, we utilize the least-mean-square (LMS) algorithm [10][12] to calculate weight values for choosing delay connections. The performance of the proposed connection algorithm is demonstrated by computer simulation.

This paper is organized as follows. In section 2, we define signal models for the switch, and an adaptive algorithm based on the LMS is discussed in section 3. In section 4, we present an implementation of the connection constraint and computer simulation is provided in section 5 to demonstrate properties of the proposed algorithm. Finally, we make a final conclusion in section 6 .

\section{Signal model for the switch}

A set of input and output signals for the $N \times N$ switch represented by the weight matrix for the SRAF can be defined by

$$
\begin{aligned}
& \mathbf{x}(k)=\left[x_{1}(k), \ldots, x_{N}(k)\right] \\
& \mathbf{y}(k)=\left[y_{1}(k), \ldots, y_{N}(k)\right]
\end{aligned}
$$

where $k$ is the discrete-time index, shown in Figure 1. Delays at the input and output of the switch can be represented by the following matrices

$$
\mathbf{D}_{x}\left(z^{-1}\right)=\left[z^{-m_{1}}, \ldots, z^{-m_{N}}\right]^{T}
$$




$$
\mathbf{D}_{y}\left(z^{-1}\right)=\left[z^{-n_{1}}, \ldots, z^{-n_{N}}\right]^{T}
$$

The switch weight matrix $\mathbf{W}(k)$ connects the elements of $\mathbf{x}(k)$ and $\mathbf{y}(k)$ such that at most there is only one nonzero element in each row and each column. Combing these definitions, the overall output can be written in terms of the input as follows:

$$
y(k)=\mathbf{D}_{y}^{T}\left(z^{-1}\right) \mathbf{W}(k) \mathbf{D}_{x}\left(z^{-1}\right) x(k)
$$

where $z^{-1}$ in this time-domain expression is the delay operator (i.e., $z^{-1} x(k)=x(k-1)$ ).

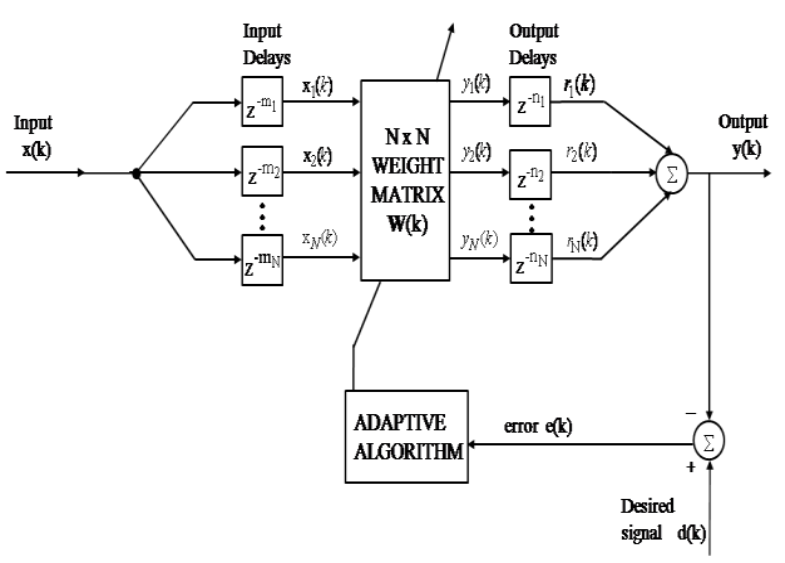

Figure 1. SRAF architecture

\section{Adaptive algorithm based on LMS}

The output error signal can be written as

$$
e(k)=d(k)-y(k)
$$

where $d(k)$ is the desired signal. The LMS algorithm for computing the weight vector is:

$$
\mathbf{w}(k+1)=\mathbf{w}(k)+2 \mu \mathbf{r}(k) e(k)
$$

where $\mu>0$ is the step-size parameter for controlling the convergence properties of the system, and with the regression vector $\mathbf{r}(k)$ given by

$$
\begin{aligned}
\mathbf{r}(k) & =\mathbf{D}_{y}\left(z^{-1}\right) \mathbf{W}(k) \mathbf{D}_{x}\left(z^{-1}\right) \mathbf{1} x(k) \\
& =\mathbf{D}_{y}\left(z^{-1}\right) \mathbf{W}(k) \mathbf{x}(k) \\
& =\mathbf{D}_{y}\left(z^{-1}\right) \mathbf{y}(k)
\end{aligned}
$$

where $\mathbf{1}=[1, \ldots, 1]^{T}$ is of size $N$.

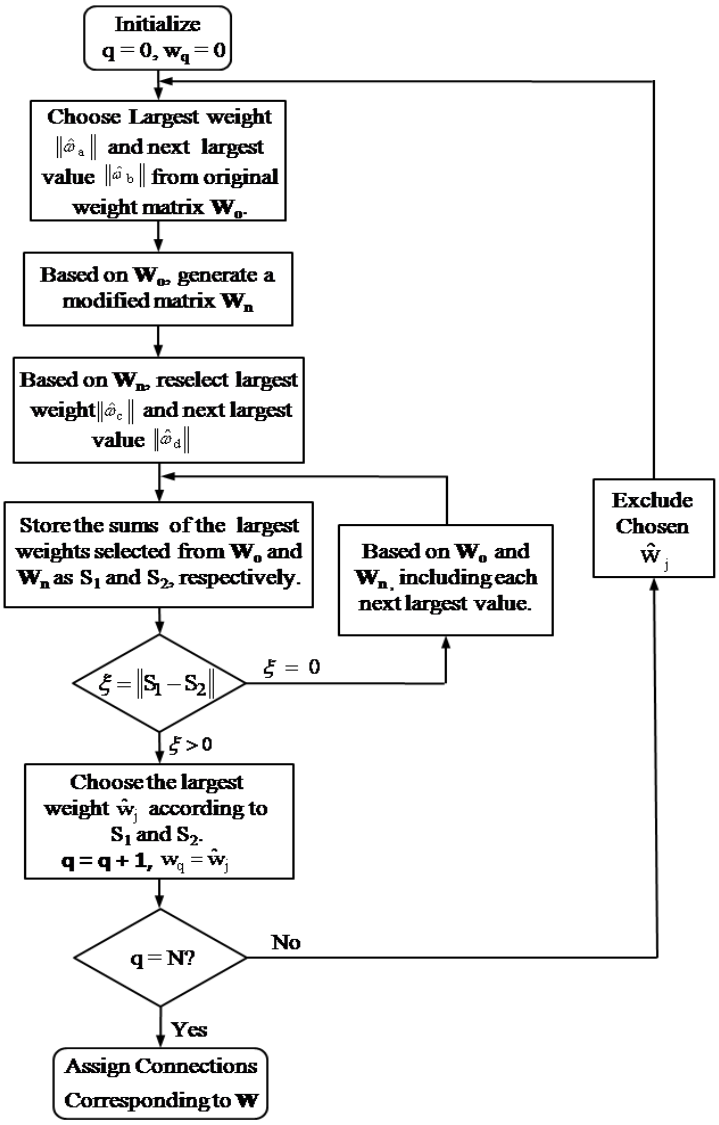

Figure 2. Algorithm flowchart for choosing the $N$ largest weights subject to the connection constraint.

\section{Implementation of the connection constraint}

In this section, we present a connection constraint algorithm to choose available weights among the calculated weights in Section 3. Although a connection algorithm based on sequentially choosing the maximum elements is investigated previously, it might not work well if the same values exist when computing the weight values. In order to solve this problem, an upgraded connection algorithm is motivated. A simple example is considered as follows:

$$
\mathbf{w}=\left[\begin{array}{ccc}
2 & 4 & 9 \\
5 & 8 & 6 \\
7 & 12 & 13
\end{array}\right]
$$

Using the initial connection algorithm, 13 [located at $(3,3)$ ], 8 [located at $(2,2)$ ], and 2 [located at $(1,1)$ ] would be chosen. Because we want to maximize the sum of the weight magnitudes, this selection is not optimal. The optimal connection is given by 12 [located at $(3,2)$ ], 9 [located at $(1,3)$ ], and 5 [located at $(2,1)]$. The proposed algorithm is represented as follows:

1) Choose the largest and next largest values (magnitudes) from those located in the different row and column of 
the weight matrix.

2) Store the sum of the two largest weights.

3) Based on the original weight matrix, generate a new weight matrix by using zero instead of the largest weight.

4) Repeat 1 and 2, and choose the largest and next largest values based on the new weight matrix.

5) Store the sum of the two largest weights selected from 4.

6) If the value in 2 exceeds that in 5 , then the largest weight from 1 is chosen. Otherwise, if the value in 5 exceeds that in 2 , the largest weight from 4 is chosen.

7) If the value in 2 equals that in 5, then based on 2 and 5 , including each next largest value, restore the sums of the largest weights, respectively, and go to 6 .

8) Continue this procedure until $N$ weights have been selected.

Figure 2 shows an algorithm flowchart for the proposed connection algorithm.

\section{Computer simulation}

Next, we describe computer simulation results of the proposed connection algorithm for implementing the filter. For the simulation, Parks-McClellan algorithm is used to generate a linear-phase FIR bandpass filter with 64 coefficients. We specified that the passband be flat over the normalized frequency range $[0.4,0.5]$ where unity represents the Nyquist frequency. The stop-bands were chosen to be zero in the range $[0,0.3]$ and $[0.6,1]$ (so that the transition bands are $(0.3,0.4)$ and $(0.5,0.6))$. The proposed connection algorithm was run for $\mathrm{M}=10,000$ input samples and with a step-size parameter of $\mu=0.001$.

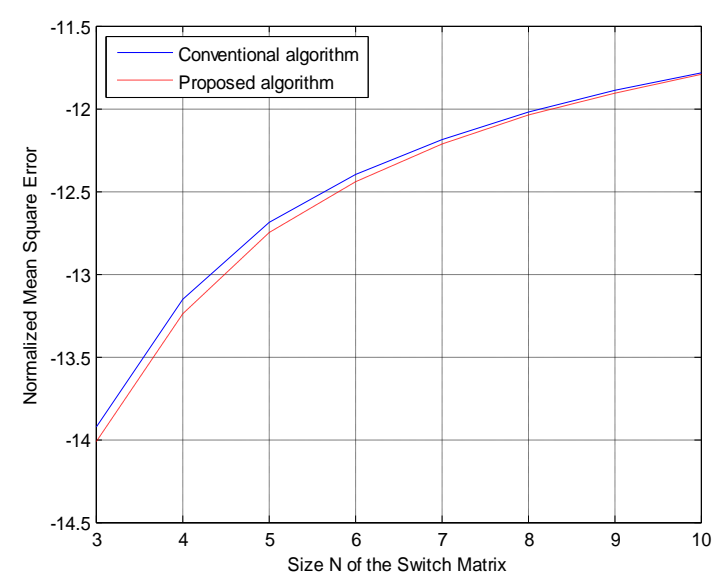

Figure 3. MSE curve for conventional and proposed connection constraint algorithms.

Figure 3 compares the mean-square-error (MSE) [13] obtained by averaging the squared error over 10,000 samples independent computer runs for the conventional and proposed connection algorithms. Observe that the dotted line (MSE of proposed algorithm) is lower than the solid line (MSE of conventional algorithm) for all matrix sizes of the switch. From the figure we observe that the proposed connection algorithm has better performance than the conventional connection algorithm, but at the expense of an increase in the computational complexity.

The impulse and frequency responses of the system are shown in Figure 4 and we can observe that the stop-band is 60$80 \mathrm{~dB}$ lower than the pass band. The resulting impulse and frequency responses are shown in Figures 5 and 6, respectively. From these figures, we observe that the converged adaptive filter has about $40 \mathrm{~dB}$ rejection capability in the stop-band for both cases, and we also find that adaptive algorithm has good performance for not only the white input signal but also the non-white input signal.

\section{Conclusions}

A sparse reconfigurable adaptive filter (SRAF) which consists of a large number of input and output delays connected together by weights is highly flexible due to its ability to choose from a wide range of delay values. In this paper, an upgraded connection constraint algorithm that can choose the better input and output delay values for implementing the connection constraints for the SRAF was described to increase the accuracy of system identification. The main idea behind the upgraded algorithm is that if the same values of summation of the weight magnitudes exist, based on previous calculation, another largest weight has to be considered for choosing the better solution. The proposed connection constraint algorithm has better performance than the conventional one, at the expense of an increase in the computational complexity. Computer simulation example was presented to illustrate the performance of the proposed connection algorithm. 

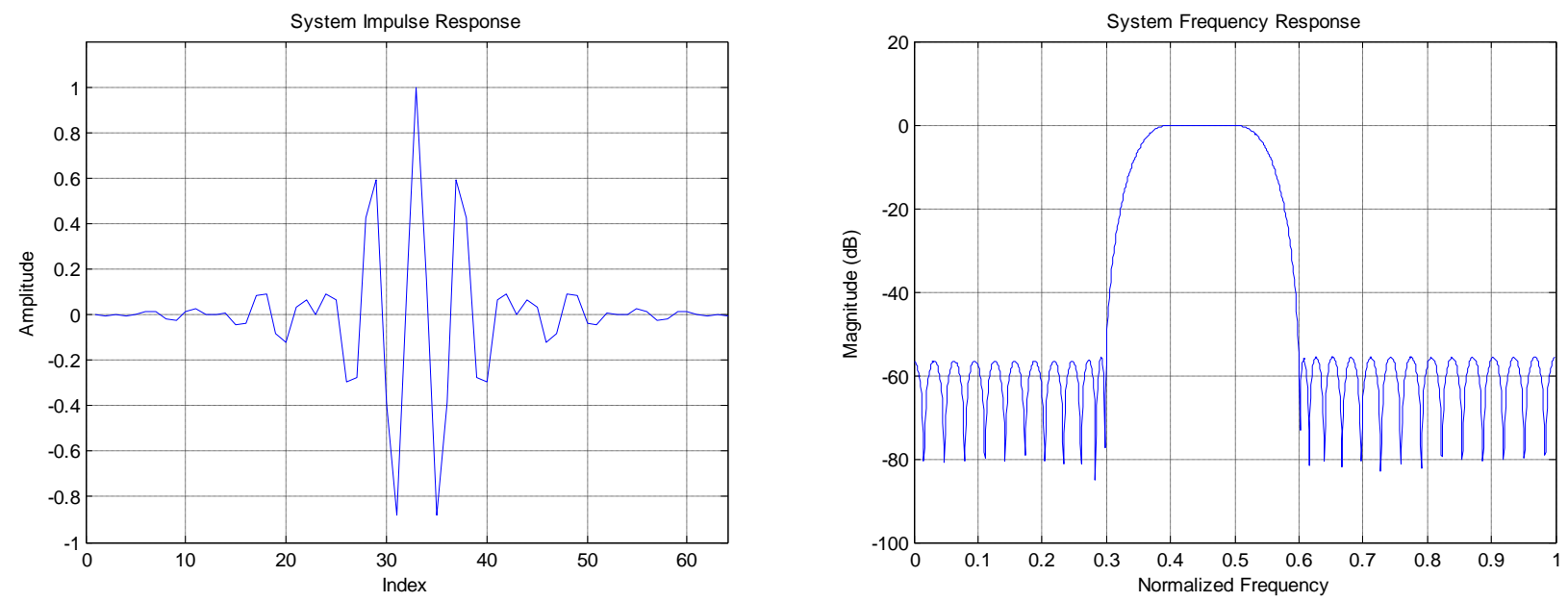

Figure 4. Impulse response and frequency response of the actual bandpass filter system with 64 coefficients.
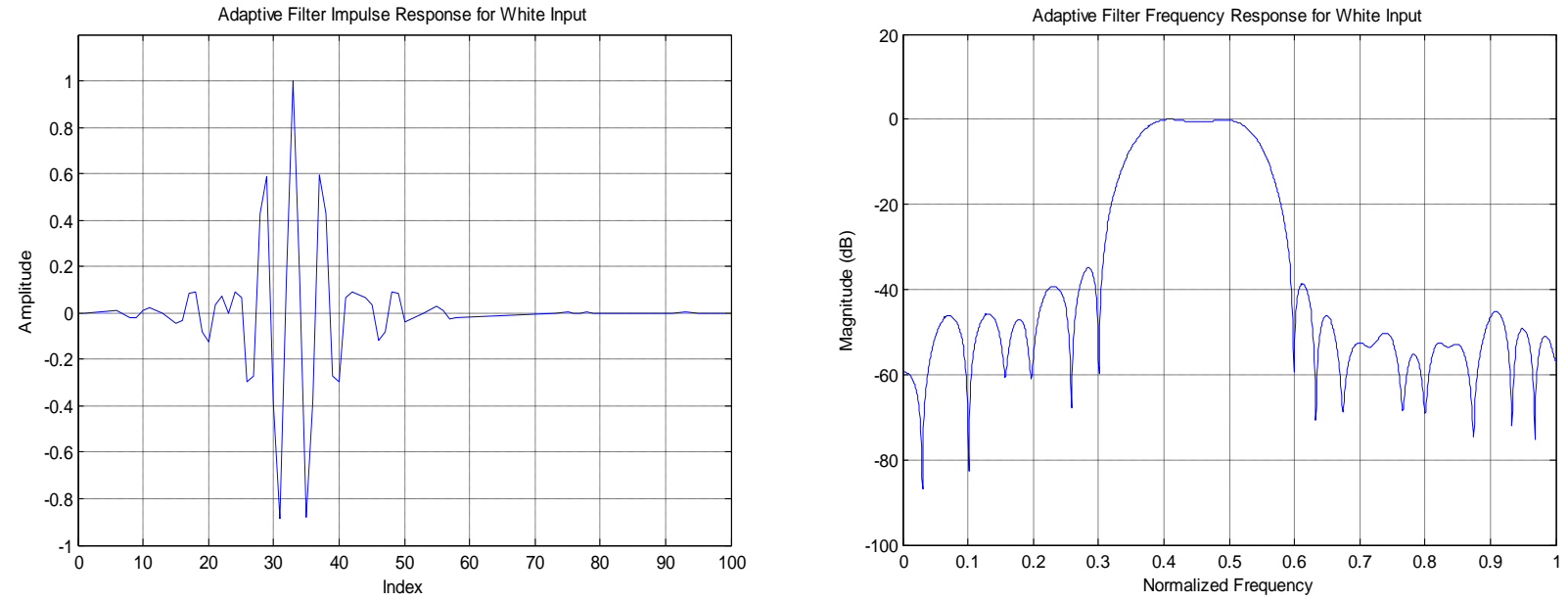

Figure 5. Impulse response and frequency response of the adaptive system with 64 nonzero coefficients for a white input signal.
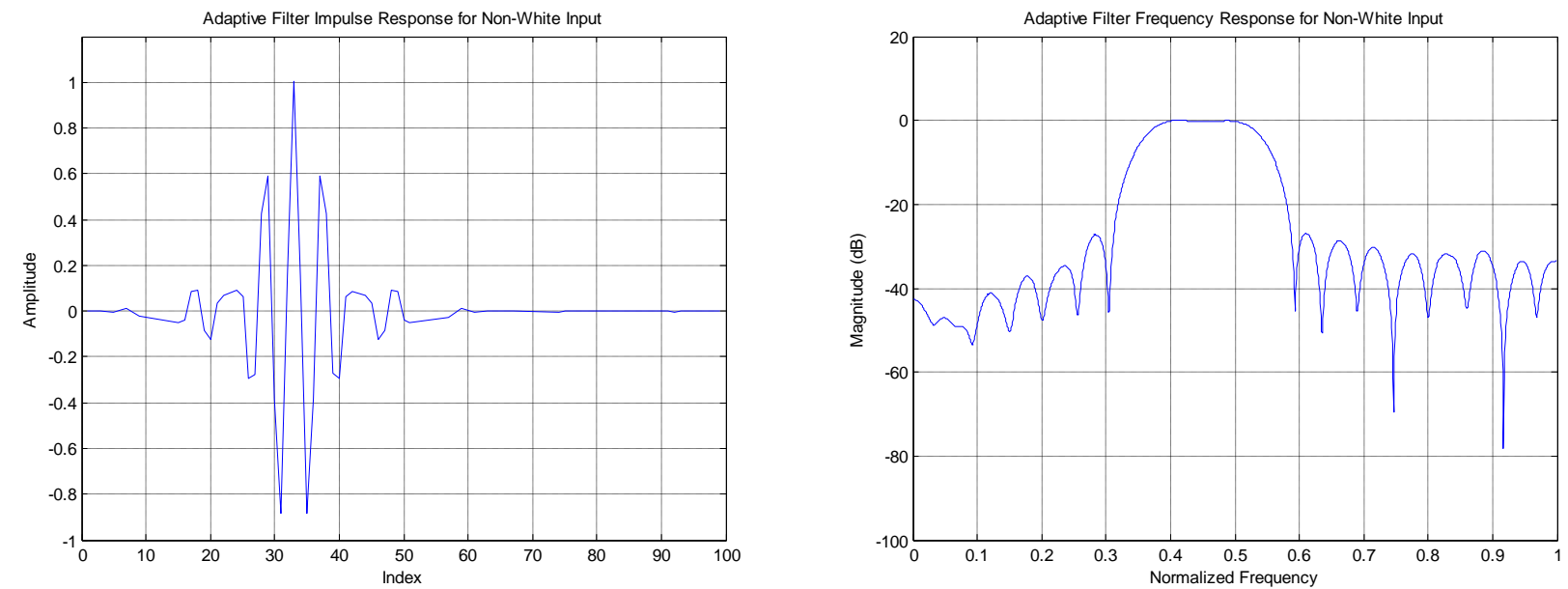

Figure 6. Impulse response and frequency response of the adaptive system with 64 nonzero coefficients for a non-white input signal 


\section{References}

[1] J. J. Shynk, J. E. Bowers, and S. Hwang, "A sparse reconfigurable adaptive filter based on a photonic switch," in Proc. 38th Asilomar Conf. Signals, Syst., Comput., Pacific Grove, CA, pp. 235-240, Nov. 2004.

[2] R. Helkey, S. Adams, J. Bowers, T. Davis, O. Jerphagnon, V. Kaman, A. Keating, B. Liu, C. Pusarla, D. Xu, S. Yuan, and X. Zheng,"Design of large, MEMS-based photonic switches," Opt. Photon. News, vol. 13, pp. 42-45, May 2002.

[3] M. Tur, J. W. Goodman, B. Moslehi, J. E. Bowers, and H. J. Shaw, "Fiber-optic signal processor with applications to matrix-vector multiplication and lattice filtering,"Opti. Lett., vol. 7, pp. 463-465, Sep. 1982.

[4] T. Yamamoto, J. Yamaguchi, R. Sawada, and Y. Uenishi, "Development of a large-scale 3-D MEMS optical switch module," NTT Tech. Rev., vol. 1, pp. 37-42, Oct. 2003.

[5] V. Kaman, X. Zheng, R. J. Helkey, C. Pusarla, and J. E. Bowers, "A 32-element 8-bit photonic true-time-delay system based on a $288 \times 2883$-D MEMS optical switch," IEEE Photon. Technol. Lett., vol. 15, pp. 849-851, Jun. 2003.

[6] X. Zheng, V. Kamen, S. Yuan, Y. Xu, O. Jerphagnon, A. Keating, R. C. Anderson, H. N. Poulsen, B. Liu, J. R. Sechrist, C. Pusarla, R. Helkey, D. J. Blumenthal, and J. E. Bowers, "Three-dimensional MEMS pho- tonic crossconnect switch design and per- formance,"IEEE J. Sel. Topics Quantum Electron., vol. 9, no. 2, pp. 571-578, Apr. 2003.

[7] Kyoung-Joo Kim, Yoon Ho Choi, Jin Bae Park, "Indirect Adaptive Control of Nonlinear Systems Using a EKF Learning Algorithm Based Wavelet Neural Network", Journal of Korean Institute of Intelligent Systems, vol. 15, no. 6, pp. 720-729, Dec. 2005.

[8] S. Hwang, J. J. Shynk, and J. E. Bowers, "A system-based adaptive algorithm for a reconfigurable photonic switch," in Proc. 39th Conf. Inf. Sci. Syst., Baltimore, MD, Mar. 2005. pp. TA8.3.1-8.3.4.

[9] S. Hwang, J. J. Shynk, T. Kang and J. E. Bowers, "Algorithms for a sparse reconfi- gurable adaptive filter and a photonic switch architecture," IEEE Transactions on circuits and systems, Regular papers, vol. 55, no. 1, pp. 347-360. Feb. 2008.

[10] B. Widrow and S. D. Stearns, Adaptive Signal Processing. Englewood Cliffs, NJ: Prentice-Hall, 1985.

[11] S. S. Narayan and A. M. Peterson. Freque- ncy domain least-mean-square algorithm. Proc. IEEE, 69(1):124-126, January 1981.

[12] Yoon-Ho Park, Se-Min Kim, "Radial Basis Function Network Based Predictive Control of Chaotic Nonlinear Systems", Journal of Korean Institute of Intelligent Systems, vol. 13, no. 5, pp. 606-613, Oct. 2003.
[13] Oh-Sung Byun and Sung-Ryong Moon, "A study on FCNN structure based on a $\alpha$-LTSHD for an effective image processing", Journal of Korean Institute of Intelligent Systems, vol. 12, no. 5, pp. 467-472, 2002.

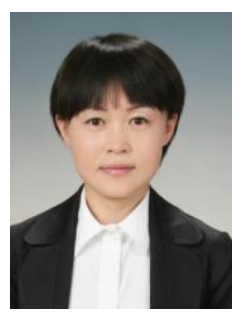

Hong Chang received the B.S. degree in Mechanical Engineering from Liaoning University of Technology, China, in 2007, and the M.S. degree in Advanced Parts and Materials Engineering from Chosun University, Korea, in 2011.

Her research interests include the adaptive algorithm and upgraded connection constraint algorithms for the SRAF.

Suk-seung Hwang was born in Seoul, Korea, and received the

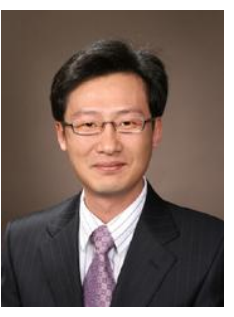
BS degree in Control and Instrumentation Engineering from Kwangwoon University, Seoul, in 1997, and the MS and PhD degrees in Electrical and Computer Engineering from the University of California, Santa Barbara (UCSB), in 2001 and 2006, respectively.

At UCSB, his interests included adaptive signal processing applied to wireless communications, interference cancelation for GPS, and adaptive algorithms for an optical switch. He was a Teaching Assistant and a Graduate Student Researcher from 1999 to 2006. From 2006 to 2008, he was a Senior Engineer with the Telecommunication R\&D Center, Samsung Electronics Company, Ltd., Suwon, Korea. At Samsung, his research interests included smart antennas and MIMO for $4 \mathrm{G}$ wireless communication systems, channel estimation, and location-based service algorithms. Since 2008, he has been with the Department of Mechatronics Engineering, Chosun University, Gwangju, Korea, where he is currently an Assistant Professor. His current research interests include adaptive signal processing for wireless communications and interference suppression, location detection technology (LDT) based on GPS and non-GPS, and wireless communications for a tire-pressure monitoring system (TPMS).

Dr. Hwang was selected as outstanding Teaching Assistant in Electrical Engineering at UCSB in 2002 and 2003.rd University

E-mail : $\underline{\text { hwangss@ chosun.ac.kr }}$ 